\title{
CONTENT-BASED IMAGE RETRIEVAL BASED ON COLOR-SPATIAL FEATURES
}

\author{
Mas Rina Mustaffa, Fatimah Ahmad, Rahmita Wirza O.K. Rahmat, Ramlan Mahmod \\ Department of Multimedia, \\ Faculty of Computer Science and Information Technology, \\ Universiti Putra Malaysia, 43400, Serdang, \\ Selangor Darul Ehsan, Malaysia \\ Email: \{masrina, fatimah, rahmita, ramlan\}@fsktm.upm.edu.my
}

\begin{abstract}
A novel technique for Content-Based Image Retrieval (CBIR) that employs both the color and spatial information of images is proposed. A maximum of three dominant color regions in an image together with its respective coordinates of the Minimum-Bounding Rectangle (MBR) are first extracted. Next, the Sub-Block technique is then used to determine the location of the dominant regions by comparing the coordinates of the region's MBR with the four corners of the center of the location map. The cell number that is maximally covered by the region is supposedly to be assigned as the location index. However, the Sub-Block technique is not reliable because in most cases, the location index assigned is not the cell number that is maximally covered by the region and sometimes a region does not overlap with the cell number assigned at all. The effectiveness of this technique has been improved using the Improved Sub-Block technique by taking into consideration the total horizontal and vertical distances of a region at each location where it overlaps. The color-spatial technique is accessed on a Query-byExample CBIR system consisting of 900 images. From the experiments it is shown that retrieval effectiveness has been significantly improved by $85.86 \%$.
\end{abstract}

Keywords: Color, Content-Based Image Retrieval (CBIR), Query-by-Example, Spatial.

\subsection{INTRODUCTION}

CBIR is an exciting and in-depth area of research, which has garnered much interest over the past few years. The relevance of visual information retrieval in many areas such as fashion and design, crime prevention, medicine, law, and science makes this research field one of the important and fastest growing in information technology. Image retrieval has come a long way where it started off with text-based retrieval. However, there are many problems associated with retrieving images based on text such as manual annotation of keywords, differences in perceptions and interpretations, and a few others. Due to this, researchers came up with CBIR where images are retrieved based on automatically derived low-level features (human vision related), middle-level features (objects related), or high-level features (semantic related). Among these features, the low-level features are the most popular due to its simplicity compared to other level of features plus automatic object recognition and classification is still among the most difficult problems in image understanding and computer vision. One of the important low-level features is color as it plays an important role in CBIR due to its robustness to complex background and independent of image size and orientation. However, where CBIR is concern, using color alone is not sufficient to characterize an image. Some images have the same color proportions but different spatial distributions. Their appearance is clearly different, so obviously we could not assume that the color distribution always suffices to represent the pictorial content of an image. For instance, regions of blue sky and ocean may have similar color histograms, but their spatial location in images might be different. Therefore in order to rectify this problem and produce a better retrieval system, regions with similar color can be easily distinguished by imposing spatial constraints.

The aim of this research is to propose a new color-spatial approach thus contributing to an effective CBIR system. The approach has achieved up to $85.86 \%$ improvement compared to the previous work. The application can later be used in areas such as photo management, cataloging for library and museum collections, art, trademark, or logo application.

The outline of this paper is as follows. Section 2 reviews the related work. Section 3 illustrates the framework of 
the content-based image retrieval. The framework for evaluation is explained in Section 4. Section 5 presents the implemented content-based image retrieval system while Section 6 discusses the experimental results. Finally, the conclusion is presented in Section 7.

\subsection{RELATED WORK}

Various CBIR systems have been developed either for commercial use or for research prototype [1-4]. Nevertheless, not many of them include the spatial location feature, which could be very useful for searching images. Furthermore, according to Stanchev [5], we are still in need of CBIR systems employing spatial location feature.

There are several color-spatial techniques that can be found. Rao, Srihari, and Zhang [6] introduced three types of Spatial Color Histogram, which are the Annular, Angular, and Hybrid Color Histogram. All of these three techniques outperform the traditional color histogram due to the spatial information included. The annular distribution density vector is calculated by counting the number of pixels with the respective color in each annular region while the angular distribution density vector is calculated by counter-clockwise counting the number of points in each angular region, starting from the principal direction. The hybrid distribution density vector of a color on the other hand is obtained by considering both the annular and angular distribution density of a color in an image. These various color-spatial histograms provide a way to explore a particular range of color in spatial domain in detail; however the computation could be quite extensive and tedious. Shih and Chen [7] used partition-based color-spatial technique where an image is divided into 100 blocks. For each block, the first three color moments of each color component of each block are extracted and clustered into several classes based on a clustering algorithm. The mean vector of each cluster is regarded as the primitive of the image. This can be considered quite a simple approach; however it is not suitable for certain images containing background occupying a large area of the image since the method only represents the image by the mean of the principal color. Similar to the technique above, Chan and Chen [8] also considered the mean value of the color component at each block. However, instead of using 100 blocks, they divided the image into $3 \times 3$ blocks. The mean value is calculated separately for each $R, G$, and $B$ color components for each block. The advantage of considering the mean value is that effects from noises in the images and the variations in sizes of images are significantly reduced. However the disadvantage is that it is easily affected by shift variants of objects in images. Other color-spatial techniques can be found in [9-13].

Prasad, Biswas, and Gupta [14] acknowledged the importance of combining color and spatial information for better retrieval result. In their work, a maximum of three dominant color regions together with its respective coordinates of the Minimum-Bounding Rectangle (MBR) in the image are obtained through Color-Based Dominant Region segmentation [15]. The location of the regions will then be determined using the Sub-Block technique [14]. The technique works by finding the distance from the region's MBR to the coordinates of the center of the location map. According to them, the cell number that is maximally covered by a region would be assigned as the location index. However, in most cases, the index assigned is usually the cell number having the highest distance from the region's MBR to the coordinates of the center of the location map instead of the cell number that is maximally covered by the region. This will result in the wrong location assigned to the region, thus it is not reliable.

Despite the huge efforts made in the last few years, research on visual retrieval is still in its infancy. In summary, there are many aspects have yet to be exploited in relation to CBIR, especially on color-spatial features.

\subsection{CONTENT-BASED IMAGE RETRIEVAL FRAMEWORK}

Fig. 1 shows the interaction between the components of the proposed CBIR system. Firstly, the collected images will go through an initialization process. During this process, the size of the image is determined. The images will then go through feature extraction component. This stage is very important, as this is when the color and the location information are extracted from the images. The color information is extracted using the Dominant Color Region segmentation [15] while the region's location is determined using the proposed Improved Sub-Block technique. The features extraction is done automatically by the CBIR system. All of the extracted information is then stored in a database. The extracted color and location information of an image is then used to create all 
possible indexes for that image. The generated indexes are stored in the index table. All of the images in the image database are processed off-line to save query-processing time. Only images that have been processed by the feature extraction component are available for querying and retrieval. During the query process, when the user has given an example of the query image, the query image will need to go through the same initialization, feature extraction, and indexing process. The indexes of the query image are compared with each of the indexes in the index table. Images with similar index as the query image are returned to the user through a Graphical User Interface (GUI). A more detailed explanation for each process can be found in the next subsection.

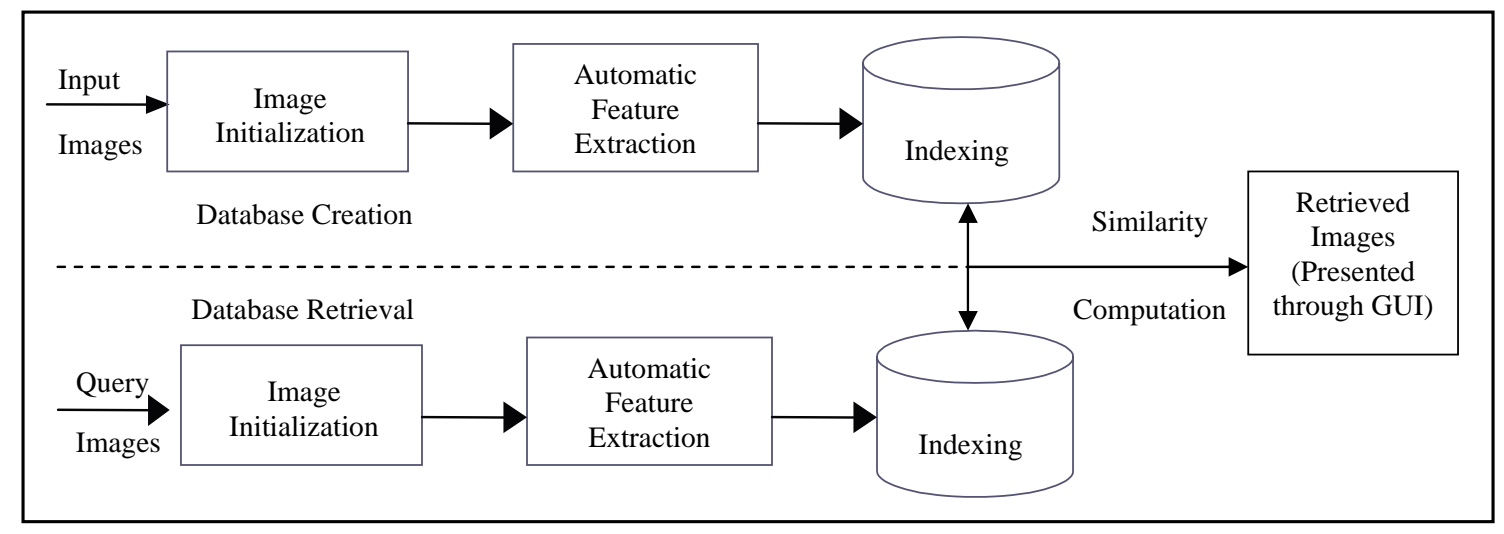

Fig. 1: Content-based image retrieval system framework

\subsection{Image Initialization}

The images are set to a size of $192 \times 128$. All of the images are in the JPEG format and RGB color space. The reason that these constraints are applied to the images is to make it suitable for this application and also during retrieval time.

\subsection{Feature Extraction}

\subsubsection{Color-Based Dominant Region Segmentation}

According to the dominant color region applied in [15], the entire $R G B$ color space is described using 25 color categories, which is summarized into a color look-up table. Image is segmented into regions according to their perceived color. It involves mapping all pixels to their categories in color space and grouping pixels belonging to the same category. A color from the color look-up table that is very near to the image pixel color is then selected and it will be stored as new color pixel in the image. This will be done using the Euclidean distance formula (the distance between the image pixel value and the corresponding color table entry is calculated. An $R G B$ component of the color look-up table is assigned to the pixel where distance is minimum). The information will be stored in a frequency table in descending order to give the dominant color regions. Region marking is done using 8Connected Neighboring Region Growing method [16]. A bounding rectangle is drawn for each selected dominant region. The area of boundary rectangle is used in determining the normalized area of the dominant region. Three regions with the biggest area will be chosen as the three most dominant regions.

\subsubsection{Improved Sub-Block Technique}

The image space is divided into nine sub-locations. Each of the sub-location is numbered from one to nine, and labeled as left top, middle top, right top, left center, center, right center, left bottom, middle bottom, and right bottom. Instead of using the distance from the region's MBR to the coordinates of the center of the location map to determine the location of a dominant region, a more effective approach is applied. The first step is to identify all sub-locations overlapped by that dominant region. Then for each of that sub-location, the horizontal edge of the dominant region is identified. The horizontal distance from the left edge to the right edge of the dominant region within that sub-location is then calculated. This process will be repeated for each of the horizontal line of the dominant region within that sub-location. The horizontal distances for the dominant region of that particular sub-location will then be added up to obtain the total horizontal distance. Next, the vertical edge of the dominant 
region within that particular sub-location is identified. The vertical distance from the top edge to the bottom edge of the dominant region within that sub-location is then calculated. This process will be repeated for each of the vertical line of the dominant region within that sub-location. The vertical distances for the dominant region of that particular sub-location will then be added up to obtain the total vertical distance. A sketch of the horizontal and vertical distance is shown in Fig. 2. Next, the overall distance of the dominant region for that particular sublocation is calculated by adding the total horizontal distance and total vertical distance. After obtaining the overall distance for each of the sub-location where the region overlaps, they will be compared and the sublocation with the highest distance will be assigned as the location index for that dominant region. The algorithm for the Improved Sub-Block technique is shown in Fig. 3.

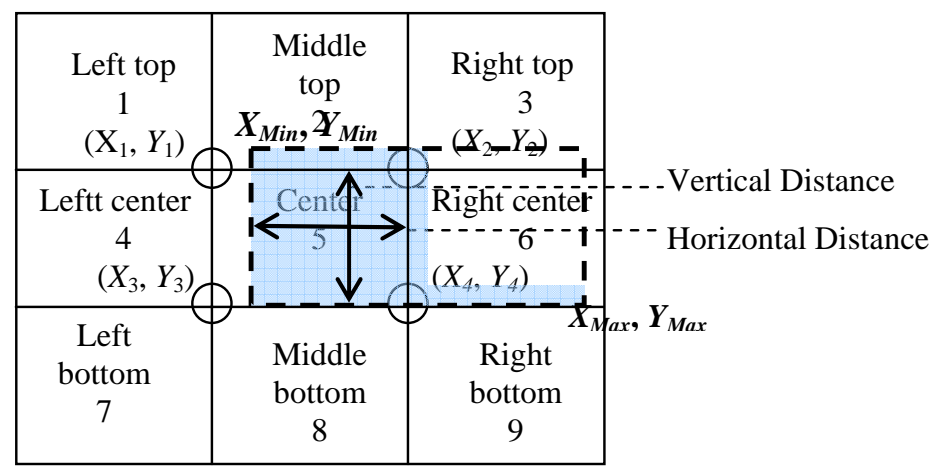

Fig. 2: A region with its horizontal and vertical distance

1. Identify the horizontal edge of the dominant region within the sub-location.

2. Calculate the horizontal distance from the left edge to the right edge of the dominant region within the sub-location.

3. Repeat for each horizontal line in that region within the sub-location.

4. Calculate the total horizontal distance for the particular dominant region within the sub-location.

5. Identify the vertical edge of the dominant region within the sub-location.

6. Calculate the vertical distance from the top edge to the bottom edge of the dominant region within the sub-location.

7. Repeat for each vertical line in that region within the sub-location.

8. Calculate the total vertical distance for the particular dominant region within the sub-location.

9. Calculate the total distance by adding the total horizontal distance and the total vertical distance within the sub-location.

10. Repeat steps 1 to 9 for each sub-location.

11. Location with the highest distance would be assigned as the location for that dominant region.

Fig. 3: Algorithm for the Improved Sub-Block technique

Note that both the total horizontal and vertical distances need to be considered in determining the location of a dominant region, instead of just considering only the total horizontal distances or total vertical distances. This is because a region may be just one line as shown in Fig. 4. 


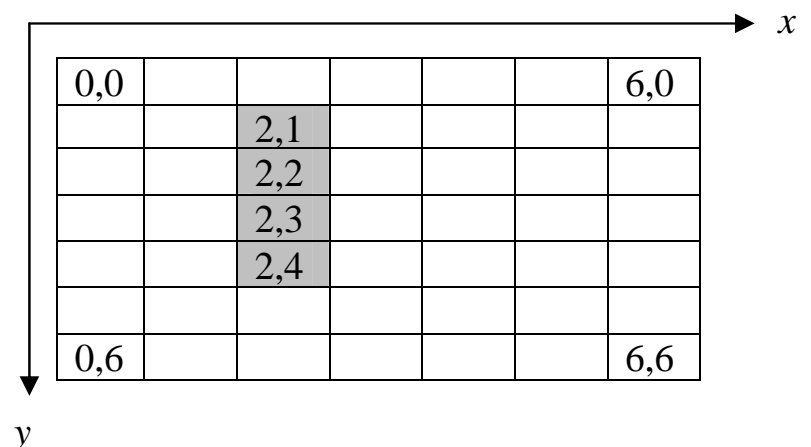

Fig. 4: A region consisting of one line

Assuming Fig. 4 is an image and each rectangle represents a pixel. If only the horizontal distances (where a horizontal distance refer to the distance from the left edge coordinate to the right edge coordinate on $x$-axis of a dominant region within a sub-location) are considered for the respective region, then the total horizontal distance is equal to zero as shown below:

$$
\begin{aligned}
\text { total horizontal distance } & =((2-2)+(2-2)+(2-2)+(2-2)) \\
& =0
\end{aligned}
$$

This should not be the case because despite being only one line, it can still be considered as one region. Therefore, by taking the vertical distances (where a vertical distance refers to the distance from the top edge coordinate to the bottom edge coordinate on the $y$-axis of a dominant region within a sub-location) into consideration, this problem can be rectified as shown below:

$$
\begin{aligned}
& \begin{aligned}
\text { total vertical distance } & =4-1 \\
& =3 \\
\text { overall distance } & =(1)+(2) \\
& =0+3 \\
& =3
\end{aligned}
\end{aligned}
$$

\subsection{Composite Color-Spatial Indexing}

The indexing technique is applied from [14]. Each region in an image is represented by a composite color-spatial index. Since three dominant regions will be considered for each image, there will be up to seven different combination indexes representing an image. All of the generated indexes will be stored in the index table. The integrated index is formed as follows:

$$
C L I=\sum_{i=1}^{n}\left[\left(25^{n-i} C_{i}\right) * 2^{10}+\left(9^{n-i} L_{i}\right)\right]
$$

where $n$ is the number of regions that will be considered within an image. As in this case, there will be up to three dominant regions within an image. $C_{i}$ and $L_{i}$ are the color and location indexes of the dominant regions respectively in decreasing order of size.

During query time, the query image will also be represented by indexes. The indexes of the query image will then be compared with the indexes of each image in the database. Those images in the database that have the same index as the query image will be retrieved as 'similar' through a GUI. 


\subsection{EVALUATION FRAMEWORK}

The experimental image database consists of 900 images from various categories like Flowers, Fruits and Vegetables, Flags, Simulated Images, Tools, and Objects. The images are obtained from various websites on the World Wide Web, Corel Photo Collection compact discs, and Image Bank compact discs. It is assumed that the image is captured using a clear background and objects in the scene are non-occluding in nature [14].

To show the practical relevance of the proposed CBIR system, retrieval experiments are conducted. The technique is evaluated based on recall and precision. A recall rate can be defined as the number of relevant documents retrieved by a search divided by the total number of existing relevant documents (which should have been retrieved). A precision rate on the other hand is the number of relevant documents retrieved by a search divided by the total number of documents retrieved by that search. Recall and precision measures have been widely used for evaluating the performance of CBIR system. This is due to its simple calculations and results obtained from these measures are easily interpreted. Apart from that, the results obtained from these measures are usually visualized through the graph representations, which will make it easier to analyze.

A total of 10 images from each image category are randomly selected and retrievals are carried out. Overall, there will be up to 60 query images selected for the whole retrieval experiments. A decision of 'relevant' for each query is done based on the Area technique. According to Oxford Dictionary [17], area is the amount of space that an object or a substance fills. In CBIR, the Area technique is applied by calculating the number of pixels in a region having the same color. Wang et al. [18] used the Area technique as part of to determine the distance between regions. An advantage of the Area technique is that it considers very detailed information of a region since it takes into account each and every pixel in a region having the respective color. Due to its high accuracy in determining the content of a region compared to other techniques, it has been chosen as the benchmark to determine whether or not an image is similar to the query image. It is also believed that the Area technique is a better benchmark compared to 'relevant judgment' by a person, which has a high tendency to be biased in describing an image. This is mainly due to the fact that different human may have a different perception when analyzing an image and only the user who knows what he or she expects as a retrieval result of a given query image. Experiments show that user judgments for the same image often differ [19]. Apart from that, user judgments are also very timeconsuming.

The total number of relevant correctly retrieved images and the total number of retrieved images obtained from each of the query performed is recorded. For each query, the recall and precision values will be interpolated to obtain the 11 standard recall-precision levels $(0.0,0.1,0.2,1.00)$. The average precision at each recall level for each image category is then calculated. They will then be represented in an average recall-precision graph where the $x$-axis of the graph is represented by the 11 standard recall levels and the $y$-axis is represented by the average precision values at the 11 standard recall levels. Since there are up to six image categories, there will be six average recall-precision graphs in total. Note that the experiments will have to be conducted for both Sub-Block technique and Improved Sub-Block technique. The technique with higher average recall-precision value is considered better compared to the other technique in terms of retrieval effectiveness.

\subsection{CONTENT-BASED IMAGE RETRIEVAL SYSTEM}

A CBIR system implementing the color-spatial technique is developed. The developed CBIR system consists of three main modules, which are the user maintenance module, category database maintenance module, and the image retrieval and performance measurement module. The user maintenance module is used to maintain users who have access to the category database maintenance module. In the category database maintenance module, the system is able to extract color and location information from an image, store the necessary extracted information into the database, and maintain the image database where new images can be added while stored images can be deleted. In the image retrieval and performance measurement module, querying and retrieval of images that have been processed by the category database maintenance module can be done. The module is able to automatically calculate the recall and precision for each query and make the relevant judgment for each of the query images based on the Area technique. The CBIR system supports Query-By-Example (QBE). Fig. 5 and Fig. 6 show the main GUI for the category database maintenance module and the image retrieval and performance measurement module respectively. The system is designed and implemented on the Microsoft Windows XP Home Edition 2000 using Microsoft Visual Basic 6.0. As for the database, Microsoft SQL Server 2000 is used. 


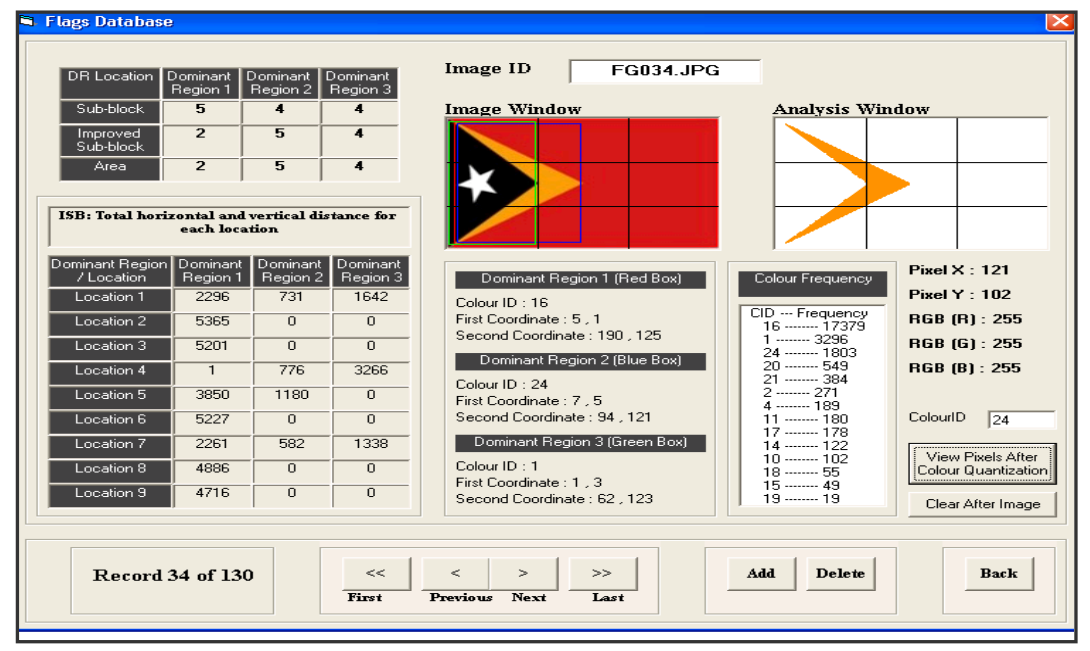

Fig. 5: Category database maintenance for 'Flags' category

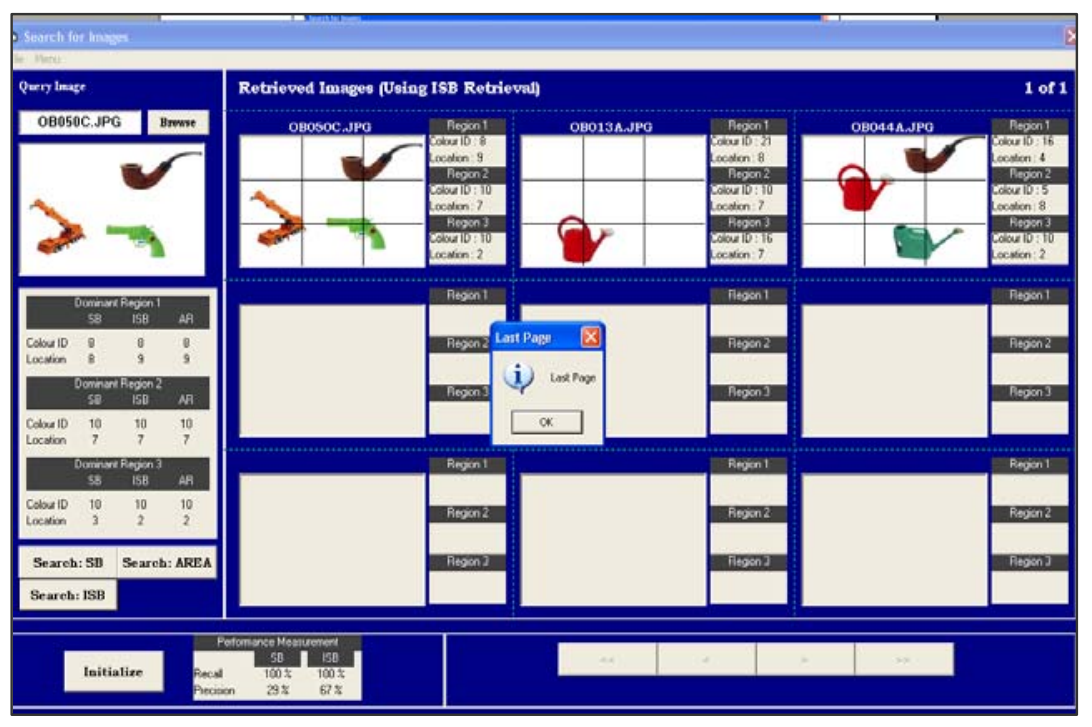

Fig. 6: A query image and the retrieval results. The recall and precision is stated at the bottom left of the interface.

\subsection{EXPERIMENTAL RESULTS AND DISCUSSION}

The improved precision achieved by the Improved Sub-Block technique according to image category is tabulated in Table 1. Figs. $7-12$ on the other hand show the graphs of the average precision of 10 query images at 11 standard recall levels for the Sub-Block (SB) and Improved Sub-Block (ISB) technique according to image category. As shown in all the average recall-precision graphs, the Improved Sub-Block technique returns a higher average precision value at most recall levels and it is also able to rank the retrieved images more accurately compared to the Sub-Block technique. From Table 1, it is shown that the Improved Sub-Block technique achieves a very high precision of about $80 \%$ for Fruits and Vegetables and Simulated Images category while the Sub-Block technique only achieves a very low precision of about $38 \%$ in average for these two categories. Hence, the precision difference for these two categories reached above $100 \%$ (precision difference $=((I S B-S B) / S B) x 100)$. This is because most objects in these categories do not fully filled the MBR area. Since the Sub-Block technique 
uses the distance from the region's MBR to the coordinates of the center of the location map to determine the location of a region, it will usually result in the wrong location being assigned to the region. In most cases, the index assigned is the cell number having the highest distance from the region's MBR to the coordinates of the center of the location map although most of the time, a different grid cell is maximally covered by a region. Even worse, sometimes a region does not overlap with the cell number assigned at all. The same goes for Flags, Tools, and Objects categories. As for the Flowers category, the precision difference between the Sub-Block and Improved Sub-Block techniques is not that significant compared to other image categories. This is because most flowers are round in shape and due to this, when a MBR is drawn on the object, the MBR area is fully filled by the object. In this kind of situation, both techniques are able to produce about the same effectiveness. From the explanation above, it can be concluded that the Improved Sub-Block technique is able to retrieve images with a higher degree of accuracy and rank the retrieved images in a correct manner far better compared to the Sub-Block technique. The improvement is mainly due to the fact that the Improved Sub-Block technique focuses too much on the MBR of an object instead of the actual object itself. While the Sub-Block technique can be effective for objects that fully filled the MBR area such as flowers without its stem, rectangular shape objects, etc., the technique fails to locate a region effectively for most of the natural, man-made, and simulated objects because most of these objects do not fully filled the MBR area. The Improved Sub-Block technique has significantly overcome the shortcomings existed in the Sub-Block technique thus improving the retrieval effectiveness of the original version by $85.86 \%$.

Table 1: Precision difference between Sub-Block (SB) and Improved Sub-Block (ISB) techniques according to image category

\begin{tabular}{|c|c|c|c|}
\hline \multirow[b]{2}{*}{$\begin{array}{l}\text { Image } \\
\text { Category }\end{array}$} & \multicolumn{2}{|c|}{ Precision } & \multirow[b]{2}{*}{$\begin{array}{c}\text { Precision } \\
\text { Difference (\%) }\end{array}$} \\
\hline & SB & ISB & \\
\hline Flowers & 0.612498 & 0.838040 & 36.823304 \\
\hline $\begin{array}{l}\text { Fruits and } \\
\text { Vegetables }\end{array}$ & 0.351525 & 0.808184 & 129.907972 \\
\hline Flags & 0.518326 & 0.879447 & 69.670632 \\
\hline $\begin{array}{l}\text { Simulated } \\
\text { Images }\end{array}$ & 0.419545 & 0.871797 & 107.795826 \\
\hline Tools & 0.374463 & 0.743638 & 98.587844 \\
\hline \multirow[t]{2}{*}{ Objects } & 0.469689 & 0.809720 & 72.394925 \\
\hline & \multicolumn{2}{|c|}{ Average } & 85.863417 \\
\hline
\end{tabular}

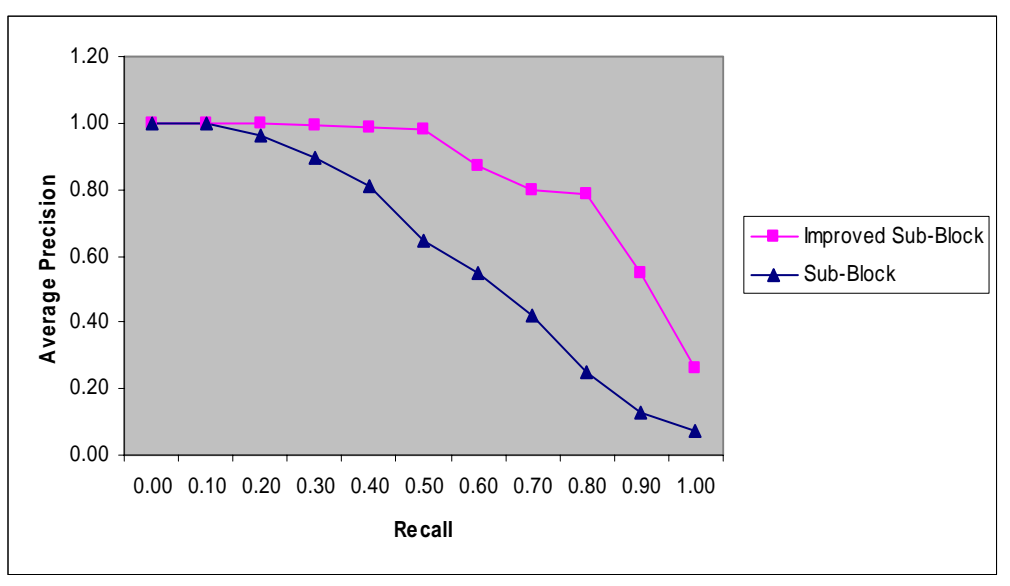


Fig. 7: Average recall and precision for SB technique versus ISB for Flowers category

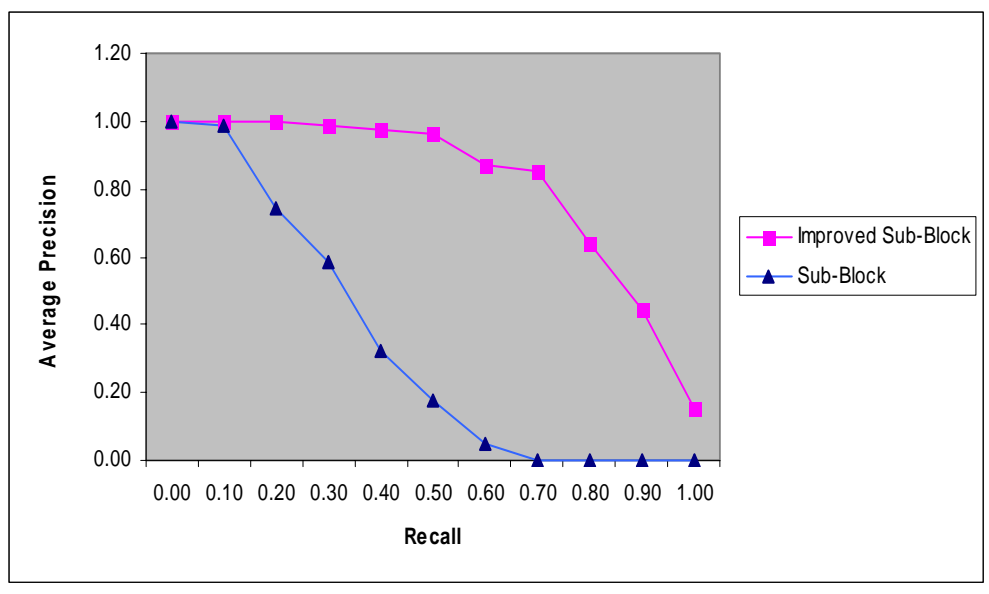

Fig. 8: Average recall and precision for SB technique versus ISB technique for Fruits and Vegetables category

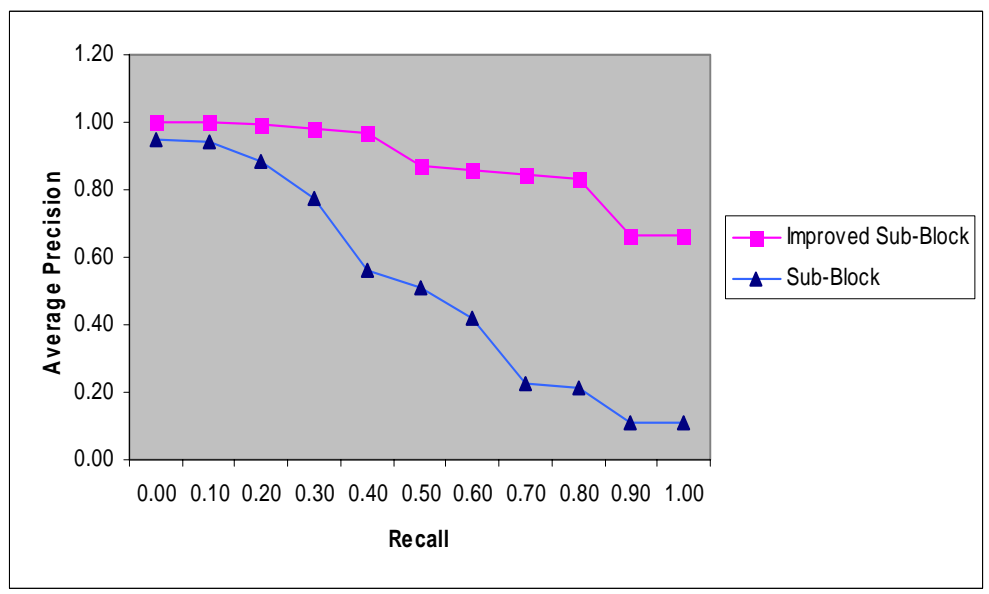

Fig. 9: Average recall and precision for SB technique versus ISB technique for Flags category

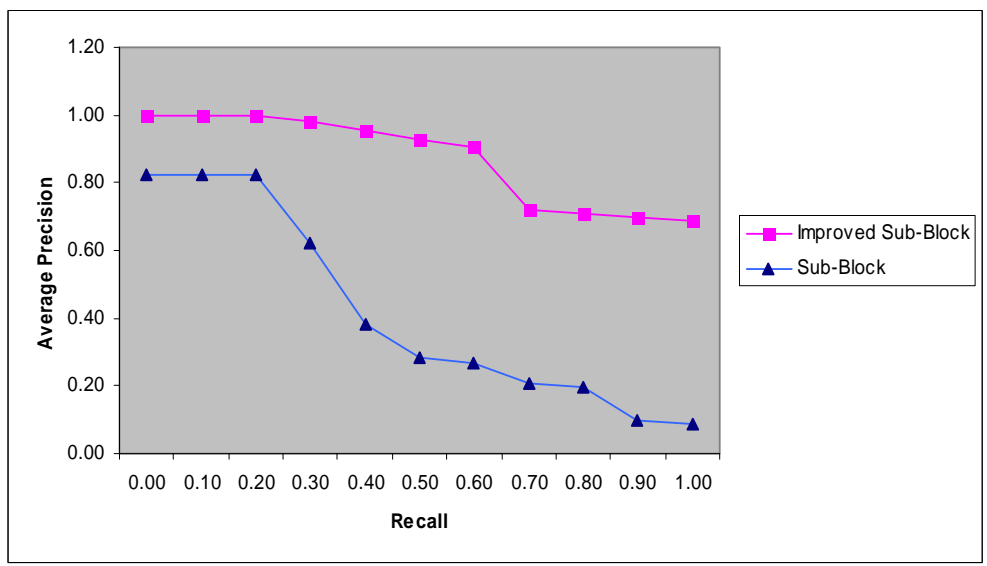

Fig. 10: Average recall and precision for SB technique versus ISB technique for Simulated Images category 


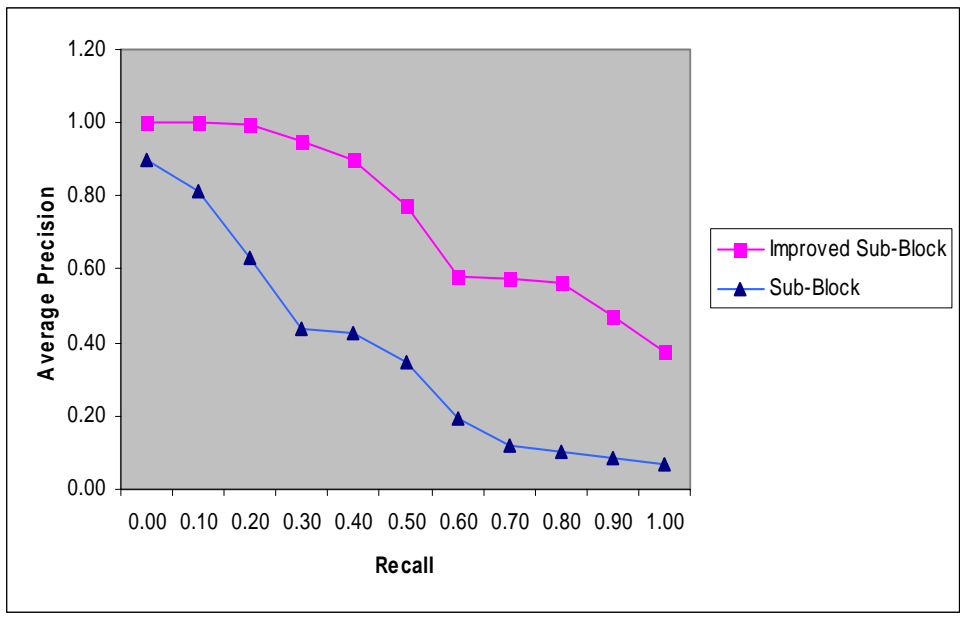

Fig. 11: Average recall and precision for SB technique versus ISB technique for Tools category

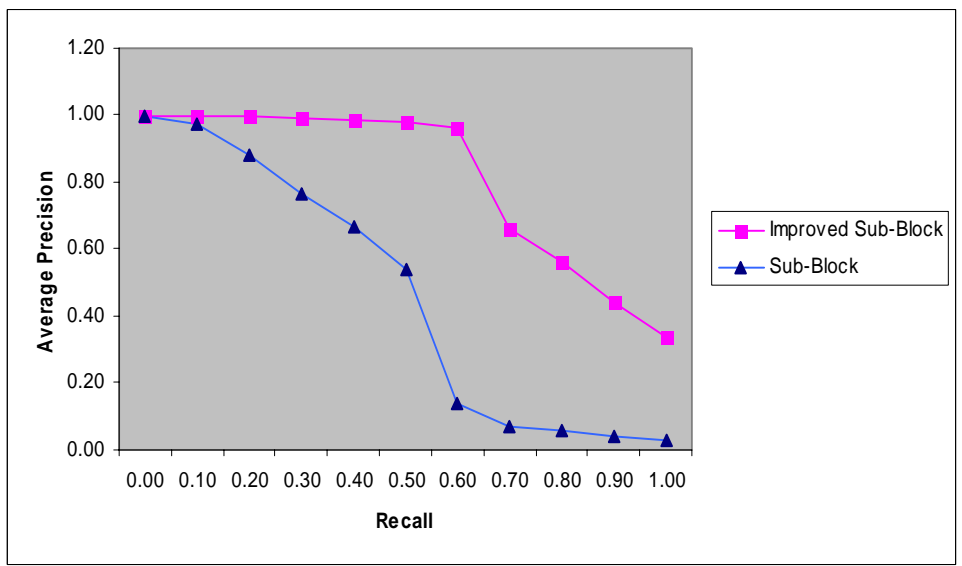

Fig. 12: Average recall and precision for SB technique versus ISB technique for Objects category

\subsection{CONCLUSIONS}

This paper focuses on developing an effective color-spatial technique for CBIR. The color information is extracted using the existing Color-Based Dominant Region segmentation while the location information is extracted using the Improved Sub-Block technique. This Improved Sub-Block technique is able to determine the relevant location of a region in an image far better compared to the original technique where the cell number that is maximally covered by the region will indeed be assigned as the location index, thus improving the retrieval effectiveness of a CBIR system by $85.86 \%$.

The color-spatial technique is implemented on a CBIR system supporting QBE. By making this refinement to the Sub-block technique, it is believed that it will be able to facilitate users in retrieving images containing objects with the same color but are located at different locations or objects having different colors but are located at the same location.

Despite showing a significant improvement in retrieval effectiveness, few extensions and refinements can be done to improve the color-spatial technique and the overall CBIR system. The color-spatial technique provides a very good retrieval performance however having a technique that is able to accommodate effectiveness and at the same time reducing a little bit of the complexity is more favorable. The Improved Sub-Block technique focuses on finding the total horizontal and vertical distances for each line of a region within a location. Future work may still focus on distance approach but does not have to consider each of the horizontal and vertical line of a region. The overall CBIR system can also be significantly improved if a technique that will be able to differentiate between 
the background and the foreground color is included allowing more complex set of images be supported by the CBIR system.

\section{REFERENCES}

[1] M. Flickner, H. Sawhney, W. Niblack, J. Ashley, Q. Huang, B. Dom, M. Gorkani, J. Hafner, D. Lee, D. Petkovic, D. Steele, and P. Yanker, "Query by image and video content: the QBIC system”, IEEE Computer, Vol. 28, No. 9, 1995, pp. 23-32.

[2] J. R. Smith and S. F. Chang, "VisualSEEk: a fully automated content-based image query system”, in Proceedings of the ACM Multimedia Conference, Boston, MA, USA, 1996, pp. 87-98.

[3] C. Carson, M. Thomas, S. Belongie, J. M. Hellerstein, and J. Malik, "Blobworld: a system for regionbased image indexing and retrieval”, in Proceedings of the Third International Conference of Visual Information and Information Systems, VISUAL '99, Amsterdam, The Netherlands, 1999, pp. 509-516.

[4] Q. Iqbal and J. K. Aggarwal, "Cires: a system for content-based retrieval in digital image libraries,” in Proceedings of the Seventh International Conference on Control, Automation, Robotics And Vision (ICARCV), Singapore, 2002, pp. 205-210.

[5] P. Stanchev, "Content-based image retrieval”, in Proceedings of the Bulgarian Computer Science Conference, CompSysTech’2001, Sofia, Bulgaria, 2001.

[6] A. Rao, R. K. Srihari, and Z. Zhang, "Spatial color histograms for content-based image retrieval”, in Proceedings of the IEEE International Conference on Tools with Artificial Intelligence, 1999, pp. 183186.

[7] J. L. Shih and L. H. Chen, “Color image retrieval based on primitives of color moments", in Proceedings of the Vision, Image, and Signal Processing, IEE, Vol. 149, No. 6, 2002, pp. 370-376.

[8] Y. K. Chan and C. Y. Chen, "Image retrieval system based on color-complexity and color-spatial features”, Journal of Systems and Software, Vol. 71, 2004, pp. 65-70.

[9] G. Pass, R. Zabih, and J. Miller, "Comparing images using color coherent vectors”, in Proceedings of the ACM Multimedia Conference, Boston, MA, USA, 1996, pp. 65-73.

[10] L. Cinque, G. Ciocca, S. Levialdi, A. Pellicano, and R. Schettini, “Color-based image retrieval using spatial-chromatic histograms”, Image and Vision Computing, Vol. 19, 2001, pp. 979-986.

[11] K. Walczak, "Image retrieval using spatial color information”, in Proceedings of the 9th. International Conference, CAIP, 2001, pp. 53-60.

[12] H. W. Yoo, H. S. Park, and D. S. Jang, “Expert system for color image retrieval,” Expert Systems with Applications, Vol. 28, 2005, pp. 347-357.

[13] J. Y. Qu and H. S. Shan, "Symmetrical color-spatial feature for medical image retrieval”, in Proceedings of the International Conference on Intelligent Information Hiding and Multimedia Signal Processing (IIHMSP '06), 2006, pp. 289-292.

[14] B. G. Prasad, K. K. Biswas, and S. K. Gupta, "Region-based image retrieval using integrated color, shape and location index”, Computer Vision and Image Understanding, Vol. 94, 2004, pp. 193-233.

[15] K. C. Ravishankar, B. G. Prasad, S. K. Gupta, and K. K. Biswas, "Dominant color region-based indexing technique for CBIR", in Proceedings of the International Conference on Image Analysis and Processing, ICIAP, Venice, Italy, 1999, pp. 887-892.

[16] R. Adams and L. Bischof, "Seeded region growing”, IEEE Transactions on Pattern Analysis and Machine Intelligence, Vol. 16, No. 6, 1994, pp. 641-647. 
[17] A. S. Hornby, Oxford Advanced Learner's Dictionary, 7th Edition. Oxford: Oxford University Press, 2005.

[18] J. Z. Wang, J. Li, and G. Wiederhold, "SIMPLIcity: semantics-sensitive integrated matching for picture libraries”, IEEE Transactions on Pattern Analysis and Machine Intelligence, Vol. 23, No. 9, 2001, pp. 947-963.

[19] D. M. Squire and T. Pun, “A comparison of human and machine assessments of image similarity for the organization of image databases”, in 10th Scandinavian Conference on Image Analysis (SCIA '97), Lappeenranta, 1997, Finland, pp.51-58.

\section{BIOGRAPHY}

Mas Rina Mustaffa received the B.Sc. degree in computer science in 2003 and the M.Sc. degree in computer science in 2006, both from Universiti Putra Malaysia, Serdang, Selangor, Malaysia. Her research interests include multimedia retrieval, computer vision, and multimedia systems.

Fatimah Ahmad received the B.Sc. degree in mathematics/computer science from University of Hartford, Hartford, Connecticut, USA, in 1986, the M.Sc. degree in computer science from Rensselaer Polytechnic Institute, Troy, New York, USA, in 1987, and the Ph.D. degree in computer science from National University of Malaysia, Bangi, Selangor, Malaysia, in 1995. She is currently an Associate Professor of the Department of Multimedia, Faculty of Computer Science and Information Technology, Universiti Putra Malaysia. Her research interests include information retrieval, multimedia computing, and natural language processing. She has published over 90 journal and conference papers. Dr. Fatimah is a member of the MNCC.

Rahmita Wirza received her M.Sc. in mathematics from University of Science, Pulau Pinang, Malaysia in 1994 and her Ph.D in mechanical engineering from University of Leeds, United Kingdom in 2000. She is currently a Senior Lecturer and the Head of Multimedia Department, Faculty of Computer Science and Information Technology, Universiti Putra Malaysia. Her research interests include multimedia computing, computer graphics applications, facial animation and surface reconstruction. She served as conference co-chair for Advanced Technology Congress (ATC'2003) and several other national conferences conducted in Malaysia. She has published over 60 journal and conference papers.

Ramlan Mahmod, biography unavailable at the time of submission. 